\title{
Understanding the variability of daily travel-time expenditures using GPS trajectory data
}

\author{
Riccardo Gallotti ${ }^{1 *}$ (D) Armando Bazzani ${ }^{2,3}$ and Sandro Rambaldi ${ }^{2,3}$
}

\author{
"Correspondence: \\ rgallotti@gmail.com \\ ${ }^{1}$ Institut de Physique Théorique, \\ CEA-Saclay, Gif-sur-Yvette, France \\ Full list of author information is \\ available at the end of the article
}

\begin{abstract}
Transportation planning is strongly influenced by the assumption that every individual has a constant daily budget of $\approx 1$ hour for his daily mobility. However, recent experimental results are proving this assumption as wrong. Here, we study the differences in daily travel-time expenditures among 24 Italian cities, extracted from a large set of GPS data on vehicles mobility. To understand these variations at the level of individual behaviour, we introduce a trip duration model that allows for a description of the distribution of travel-time expenditures in a given city using two parameters. The first parameter reflects the accessibility of desired destinations, whereas the second one can be associated to a travel-time budget and represents physiological limits due to stress and fatigue. Within the same city, we observe variations in the distributions according to home position, number of mobility days and a driver's average number of daily trips. These results can be interpreted by a stochastic time-consumption model, where the generalised cost of travel times is given by a logarithmic-like function, in agreement with the Weber-Fechner law. Our experimental results show a significant variability in the travel-time budgets in different cities, and for different categories of drivers within the same city. This explicitly clashes with the idea of the existence of a constant travel-time budget and opens new perspectives for the modelling and governance of urban mobility.
\end{abstract}

Keywords: human mobility; individual travel behaviour; duration model; travel time budget; time perception; value of time; Weber-Fechner law

\section{Introduction}

Recently, human mobility has been extensively studied using data on individual trips provided by the information-communication technologies [1-7]. In mobility-related decisions, travel time appears as a natural cost function, since it represents a limited resource used for performing daily activities [8]. The concepts of Travel-Time Expenditure (TTE, the daily amount of time spent traveling) and Travel-Time Budget (TTB, the average daily amount of time that people make available for mobility [9]) have been introduced by transportation planners to model the mobility demand and to explain some of the features characterising urban mobility [10]. Travel-Time Expenditure and Budget are more comprehensive quantities than the commuting time from home to work and back between home and work, and the related concept of Marchetti's constant [11, 12]. Indeed, this second per-

(c) 2015 Gallotti et al. This article is distributed under the terms of the Creative Commons Attribution 4.0 International License (http://creativecommons.org/licenses/by/4.0/), which permits unrestricted use, distribution, and reproduction in any medium, provided you give appropriate credit to the original author(s) and the source, provide a link to the Creative Commons license, and indicate if changes were made. 
spective is limited to the journey-to-work mobility and thus excludes a large fraction of the individuals' mobility demand associated to amenities.

The existence of a Travel-Time Budget is assumed on the basis of the behavioural hypothesis that people spend a fixed amount of time available on traveling [13]. The extreme interpretation of Travel-Time Budget as a universal constant stable in space and time is still sustained and very influential in urban planning. Indeed, if Travel-Time Budget is constant, any investments in better infrastructure would not reduce daily travel times (and possibly, through that, polluting emissions) since it would only create new induced travel demand [14]. Most of the empirical results on Travel-Time Budget are determined as average values from large travel surveys. At a disaggregate level, however, Travel-Time Expenditures appear strongly related to the heterogeneity of the individuals, to the characteristics of the activities at destinations and to the residential areas [13]. Aggregated results suggest that the average amount of time spent traveling is constant both across populations and over time: approximatively 1.0-1.1 h per day [15]. Despite the gains in average travel speed due to infrastructural and technological advances in the past decades, Travel-Time Expenditures appear more or less stable or even growing [16-18]. This growth can be associated to the super-linear relationship between a city's population and the delays due to congestion [19].

In Italy, Global Positioning System (GPS) devices are installed in a significative sample of private vehicles for insurance reasons. The initial and the final points of each trajectory are recorded, together with the path length and some intermediate points at a spatial distance of $2 \mathrm{~km}$ or at a time distance of 30 seconds. These data allow a detailed reconstruction of individual mobility in different urban contexts [20] and measure the elapsed of time during mobility [21].

In this paper, we explore the statistical features of Travel-Time Expenditures related to private mobility, both from an aggregate and individual point of view. Our goal is to point out some of the factors influencing travel demand by means of new specific measures, which describe differences among cities. The statistical analysis of empirical data points to the existence of a universal law underlying the distributions of Travel-Time Expenditures, which highlights the nature of time constraints in vehicular mobility. This result allows us to observe in detail the differences in daily travel demand for different cities, challenging the idea of a constant Travel-Time Budget and pointing out the important role of accessibility [22].

\section{Assumptions}

Previous empirical observations on different data-sources [20,23-26] have shown out that the TTE probability distribution $p(T)$ (see Table 1 for a list of notations), associated to a single mean of transportation, is characterised by an exponential tail

$$
p(T)=\beta^{-1} \exp (-T / \beta), \quad \text { for } T>1 \text { hour, }
$$

where $\beta$ is a fit parameter. Our analysis confirms the universal character of the exponential behaviour for the TTE empirical distribution and points out relevant differences among the considered cities (see Figure 1).

As it is well known from Statistical Mechanics, the exponential distribution (1) can be derived from the Maximal Entropy Principle under some minimal assumptions [20]. More 
Table 1 List of notations

\begin{tabular}{lcc}
\hline Quantity & Notation & Abbreviation \\
\hline Daily travel-time expenditure & $T$ & TTE \\
Daily travel-time budget & $\beta$ & TTB \\
Accessibility time & $\alpha$ & - \\
Single trip travel-time & $t$ & - \\
\hline \hline Function & Notation & Abbreviation \\
\hline Probability density of $x$ & $p(x)$ & PDF \\
Cumulative density of $x\left(\int^{x} p\left(x^{\prime}\right) d x^{\prime}\right)$ & $P(x)$ & CDF \\
Survival function $(1-P(x))$ & $S(x)$ & - \\
Hazard function $(d S(x) / d x)$ & $\lambda(x)$ & - \\
Conditional probability of $x$ given $y$ & $\pi(x \mid y)$ & - \\
\hline
\end{tabular}
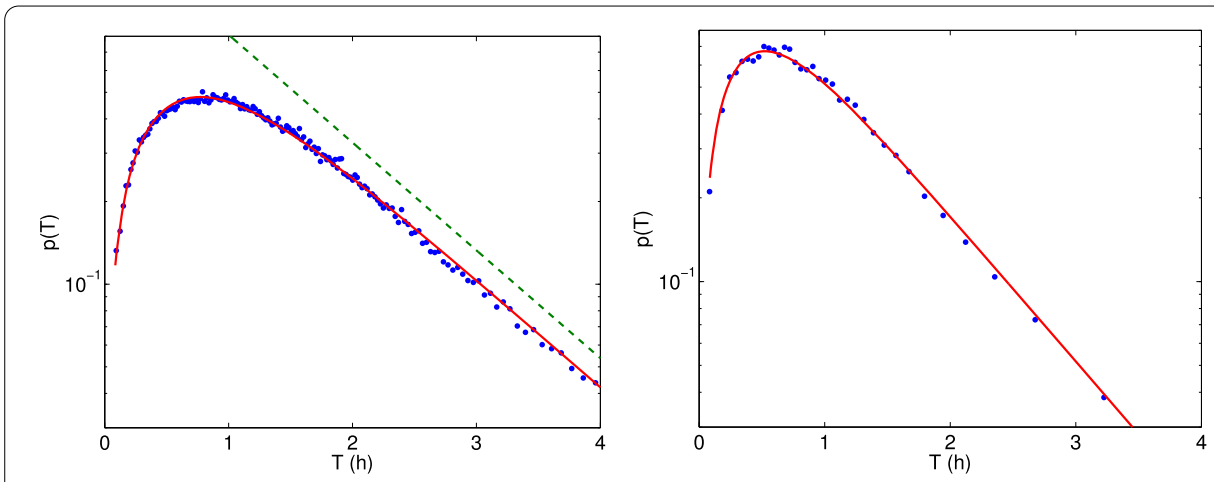

Figure 1 Travel-Time Expenditure distributions. (Left) Travel-Time Expenditure (TTE) distribution for the city of Naples $(\approx 1$ million inhabitants). The empirical probability density $p(T)$ (dots) is correctly interpolated by the curve (6) (solid line) with $\alpha=0.61 \mathrm{~h}, \beta=1.11 \mathrm{~h}$ and $R^{2}=0.99$. The dashed line, shown as a guide to the eye, represents an exponential decay with a characteristic time $\beta$. (Right) TTE distribution for the city of Grosseto ( $\approx 80,000$ inhabitants). The interpolation with the curve (6) is successful also for smaller cities like Grosseto: in this case the parameter values are $\alpha=0.38 \mathrm{~h}, \beta=0.83 \mathrm{~h}$ and $R^{2}=0.99$.

precisely, one assumes (i) existence of an average finite TTE for the considered population and (ii) the statistical independence of the behaviour of each individual. Under the constraint that the average TTE is finite, we have the same probability of observing any microscopic configuration which associates a TTE to each individual. The parameter $\beta$ defines the average time scale that limits the individual TTE and we will show that this is a characteristic of each city. Therefore, we propose to associate the concept of TTB to the value $\beta$ which characterises the exponential decay of the daily travel-time distribution. However, the Eq. (1) does not give information on the dynamical processes underlying the human mobility which produces the distribution. We take advantage from the dynamical structure of the GPS data to propose a duration model (see Section 5.2) that seems to be endowed with universal features with respect to the considered cities. The essential hypotheses at the bases of the duration model are: (i) it exists a TTB; (ii) the individual decision to continue the mobility for a time $\Delta T$, after a TTE $T$, is the realisation of an independent random event whose probability decrease proportionally to $\Delta T$.

\section{Results}

\subsection{The variability of Travel-Time Expenditures}

The average value of TTE does not give a sufficient insight on the statistical features of the distribution $p(T)$. For each city, the statistical features of the distribution $p(T)$ turn 
Figure 2 Values of $\alpha$ and $\beta$ for the 24 cities studied. The boxes represent $95 \%$ confidence intervals obtained with a bootstrap, for empirical best-fits for the model parameters. The differences we observe in the accessibility time $\alpha$ and in TTB $\beta$ are thus significant and uncorrelated $(r=0.09)$. Both timescales are weakly correlated with the city's population $(r=0.20$ for $\alpha$ and $r=0.40$ for $\beta$ ).

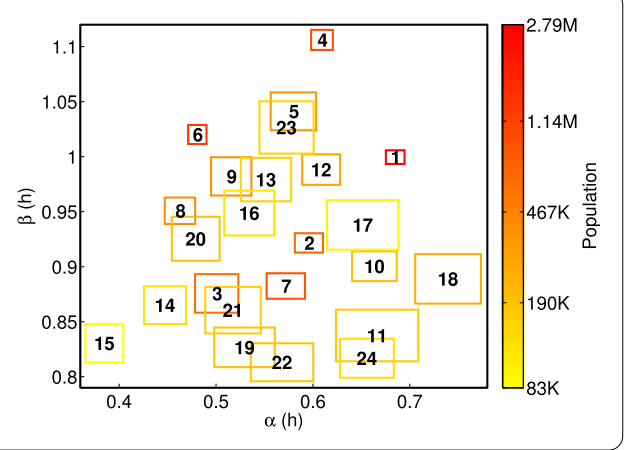

out to be characterised by the two time scales $\alpha$ and $\beta$. In the duration model, after a characteristic time $\alpha$, the choice of going back home or proceeding with further extra traveling is limited by the available TTB, whose average value is quantified by the time scale $\beta$. $\alpha$ therefore represents the average time under which the use of a private car seems to be not convenient.

We focus our study on 24 Italian cities where we have a large statistic of users. The values of $\alpha$ and $\beta$ are estimated from a best fit for $S(T)$ with Eq. (S6) (which is equivalent to fitting the CDF). The results are displayed in Figure 2 and reported in the Table S1. Two examples are also proposed in Figure 1 . The two parameters are independent, with a Pearson correlation coefficient $r=0.09 . \beta$ falls in the interval 0.8-1.1 h, which is reasonably consistent with the values reported in the literature [15]. Nevertheless, the differences we observe among cities are statistically significant, as the $95 \%$ confidence intervals for the fits, estimated with a bootstrap, are $\leq 0.02 \mathrm{~h}$. Therefore, our results clearly clash with the concept a constant TTB.

Since the values of $\beta$ are moderately correlated with the number of inhabitants of the municipality $(r=0.40)$ or population density $(r=0.49)$, some of this variability is dependent on the city population $[19,27]$. The accessibility time $\alpha$ is only weakly correlated with city population $(r=0.20)$ and not correlated $(r=0.03)$ with population density, and falls in the interval 0.3-0.8 $\mathrm{h}$. The confidence intervals for the fits are $\leq 0.04 \mathrm{~h}$, granting that we have significant differences in accessibility time among cities. The general picture, displayed in Figure 2, shows that, if one has appropriate data sources to characterise the daily mobility of a single city, one needs the knowledge of both parameters. Under this lens, the variability of TTE is manifest and can be observed in both the ramping part (characterised by $\alpha$ ) and the tail (characterised by $\beta$ ) of the distribution.

\subsection{Disaggregate analysis: the case of Milan}

Macroscopic statistical laws might depend on the details of the microscopic dynamics. Their extension down to the interpretation of the individual behaviour is therefore under debate [28]. Nevertheless, we believe that the universal character inherent to the concept of TTB could be an individual property. To support this statement, we consider here a disaggregate analysis of the GPS mobility data suggesting that our results might be extended to the individual level. A limitation of this analysis comes from the short time considered in our dataset. Indeed, it refers only to a single month of mobility, a period probably too short to infer a definitive conclusion on our hypothesis.

We study the case of the city of Milan, the largest city in North Italy with $\approx 1.3$ millions inhabitants (dash line in Figure 5(left) and labeled 6 in Figure 2). We start by verifying that 


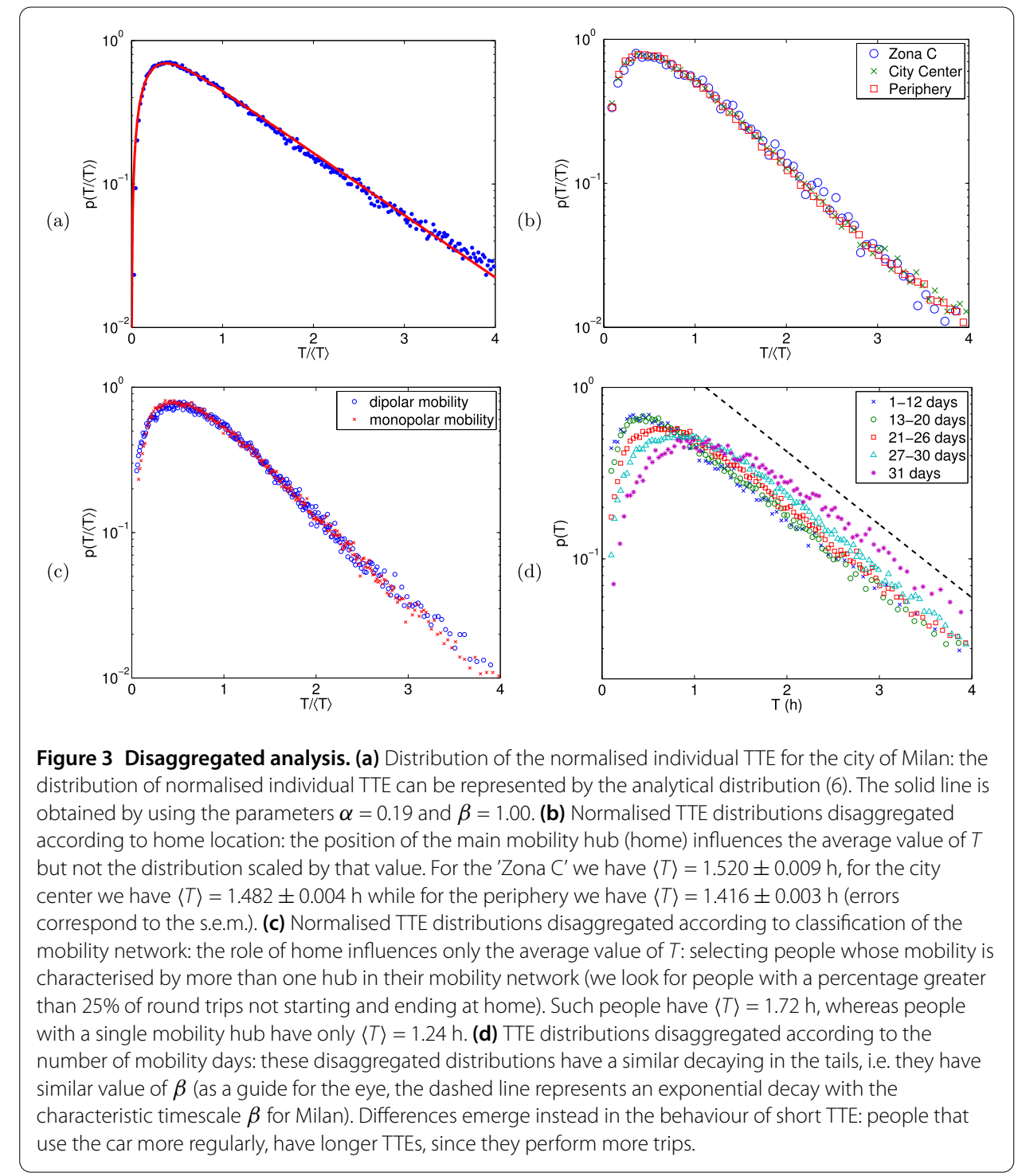

the shape of TTE distribution $p(T)$ is a property of each single individual. Using the GPS data, the heterogeneity of the population can be quantified by considering the distribution of the average individual TTE $\langle T\rangle$ empirically computed from the individual daily mobility. To compare different individuals, we normalise each TTE value by the corresponding individual average. In Figure 3(a), we show that the distribution of the normalised individual TTE $p(T /\langle T\rangle)$ is still very well fitted by the analytical curve (6). Therefore we conjecture that $\langle T\rangle$ contains the relevant information to explain the individual heterogeneity and the distribution (6) has an universal character that extends up to the individual level.

An individual disaggregation, according to the home location or characteristics of the mobility network, confirms the previous hypothesis (see Figures 3(b) and (c), and Additional file 1 for further details). Thus, the heterogeneity is mainly determined by the average value $\langle T\rangle$ evaluated within each class. However, we find that $\langle T\rangle$ is longer for:

(i) people living in the city center ( $\approx 8 \%$ longer than for people living in the periphery), a result consistent with what was found in Ref. [18] for the city of Sydney; 
conversely, people in the periphery tend to make $\approx 0.5$ trips more per day and $\approx 3.3$ days more of mobility in average;

(ii) people performing many round trips (A-B-A patterns) not involving home.

The last criterium points to the existence of a second center of daily activity and allows to separate individual mobility networks into mono-centric and polycentric ones [29].

Our empirical data suggest that people with a polycentric mobility (who have more than one mobility hub) have greater $\langle T\rangle$ than people whose round trips start and end at home. However, if we classify the individuals according to the number of days in which they used the car, the TTE distributions differ when we consider small $T$ values (see Figure 3(d)). Even if the exponential tail of the distributions does not change significantly, there is a tendency to under-express the short values of $T$ for users who regularly carry out their daily mobility by car. Our duration model associates this to a larger value of $\alpha$ and therefore the need in average of longer times to accomplish the necessary tasks of the day. In summary, people who take the car more often also need to drive more, yet maintaining a similar TTB. This is confirmed by considering the number of trips $n$ that are accomplished in a day. The average number of daily trips grows from 4.2 , for people who drove 1-12 days up to 7 for the class of users who drove all the 31 days (see Additional file 1). This result clearly links the value of the accessibility time $\alpha$ to the need of accessing to the desired destinations by car. Drivers who experience better accessibility do not need to use the car every day, and when they do they can also drive less. In the following, we show that these differences can be linked to a different value of time for users performing more trips.

\subsection{Evidence of a log-perception of travel-time costs}

Finally, we propose a time consumption model linking the duration model with the individual behaviour. This model permit to shed light on how individuals organise their mobility and allows for an interpretation of the empirical observations compatible with a logarithmic perception of the time cost of a trip (see Section 5.3). The individual decisions at the base of TTE are modelled as a stochastic process, which is consistent with the assumptions of the duration model and with a possible logarithmic perception of the cost $\Delta T$ of a trip, analogous to the Weber-Fechner psychophysical law [30]. The same model does not reproduce the empirical observations, assuming a linear time perception. This result has been confirmed with a Monte Carlo stochastic decision model, based on the same premises (see Figure 4(left) and Additional file 1). This model assumes a logit curve [31] in the decision model of the binary choice of interrupting the daily mobility after a certain trip and we could fit the TTE distributions in all cities with great precision $\left(R^{2}>0.986\right)$.

The existence of simple universal dynamical models for empirical TTE distribution allows to introduce a few observables that point out relevant differences among cities. One of those parameters can be associated to the (logarithmic) value of time (see Additional file 1). This suggests relations between the presence of mobility infrastructures and/or the socio-economic characteristics of a city, and the features of the empirical TTE distribution. These relations could be useful for urban planners to build governance policies for mobility.

\section{Discussion}

In our analysis, based on a large GPS database containing information on single vehicle trajectories in the entire Italian territory, we show that the empirical distributions for the 

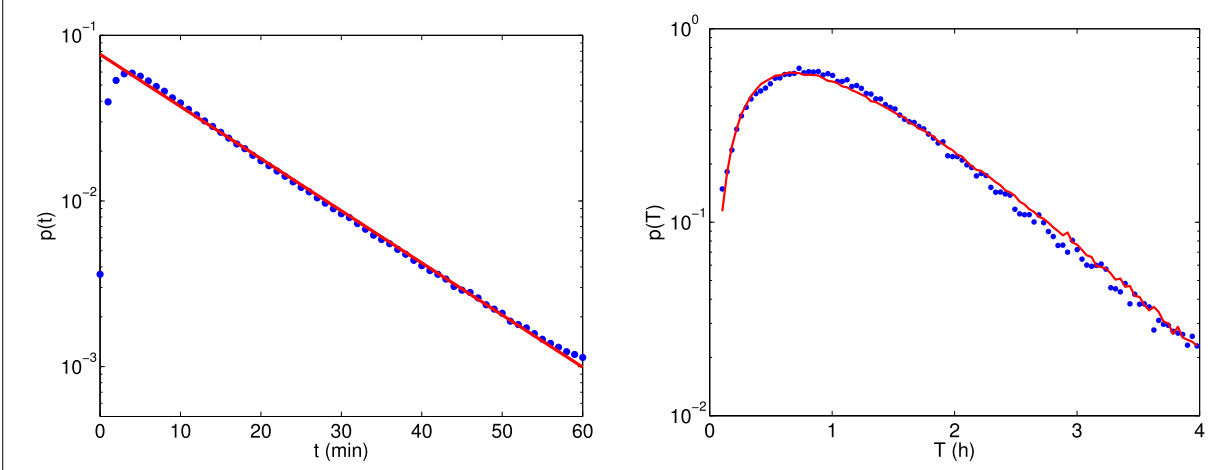

Figure 4 Travel-time distribution and stochastic decision model. (Left) The travel-time distribution $p(t)$ in Milan (dots) compared with an exponential interpolation (solid line). The under-expression for short travel-times, with $t<4$ min could be a consequence of the characteristic GPS measurement time and does not affect the time scale $\langle t\rangle$. The results are consistent with the exponential fit of the tail. (Right) Comparison between the empirical TTE $p(T)$ distribution in Turin (dots) and the best fit distribution provided by our stochastic decision model, using a logistic threshold function (solid line, see Additional file $1, R^{2}=0.99$ ).

daily Travel-Time Expenditures in different cities can be modelled by a single distribution. This distribution is function of two time scales: $\alpha$ and $\beta$. The time scale $\alpha$ measures a minimal mobility time associated to the use of private cars in a given city, whereas the limit value $1 / \beta$ of the hazard function $\lambda(T)$ as $T \gg 1$ is associated to the concept of Travel-Time Budget. In our opinion, $\alpha$ is a good measure of the average accessibility [22] of a city. Lower values of $\alpha$ (i.e. higher accessibility) mean a better proximity to useful locations and less time and trips needed for carrying out the daily mobility. We remark that if one considers Italian cities of different size and socio-economical conditions, the shape of the distribution appears to be endowed by a universal character where the only changes observed are the values of $\alpha$ and $\beta$.

Also the distribution $p(T /\langle T\rangle)$ has a universal character. This suggests the existence of a behavioural model for the urban mobility that mimics the individual decision mechanisms. As a consequence, the statistical properties pointed out by the distribution (6) are traits of the individual behaviour and the aggregated probability distribution for a city is averaging over the individual heterogeneity in the values of $\alpha$ and $\beta$ across the population. However, in the disaggregated analysis of GPS data at individual level, we find significant differences in the average Travel-Time Expenditure for different categories of drivers. In particular, drivers who use their car more often have higher values of $\alpha$ even if their $\beta$ is approximatively the same (see Figure $3(\mathrm{~d})$ ). This is another confirmation of our interpretation of the parameter $\alpha$ as a measure of accessibility, because who has the worst accessibility to public transport facilities or to the desired destinations is forced to use the private vehicle over wider range of travel-times.

To interpret these results, we propose a simple decisional model, which assumes the existence of a mobility energy (the daily travel-time) and a log-time perception of the traveltime cost for a single trip. These results are also consistent with the Benford's empirical distribution of elapsed time during human activities [20] and Weber-Fechner psychophysical law [32]. Using a Statistical Mechanics point of view, the Travel-Time Expenditure $T$ plays the role of energy in a model of the individual urban mobility based on a generalised utility function. However, one cannot simply define the trip duration $\Delta T$ as a mobility cost, because the data suggest that this perceived cost seems to decreases as the daily 
travel time $T$ grows. A time consumption model that assumes a scaling cost $\propto \Delta T / T$ (i.e. a law of relative effect [33]), corresponding to a logarithmic preference scale [30], is able to reproduce the statistical properties of the empirical observations. As a direct application of this result, we are able to suggest the use of a nonlinear relationship for the value of time in the activity-based modeling of human mobility.

At city-aggregate level, we observe that for every city the average Travel-Time Expenditure $\langle T\rangle$ is greater than the Travel-Time Budget $\beta$, because short values of $T$ are statistically under-expressed [23]. This could reflect both the fact that the individual mobility demand is hardly satisfied after short travel-times, and the disadvantage using a private car for short times. Both $\alpha$ and $\beta$ are needed to fully understand the Travel-Time Expenditures in a city. A direct application of the approach proposed permits to highlight the differences in the travel-time expenditures among cities and classes of individuals. In particular, we clearly observe a variability in the Travel-Time Budget $\beta$ among cities. The dependency upon population density and the differences observed in the disaggregate analysis explicitly clash with the idea of the existence of a fixed Travel-Time Budget.

Our results intend to nourish the discussion against this old paradigm of a constant Travel-Time Budget, which dangerously suggests that is not possible to reduce travel times, and therefore $\mathrm{CO}_{2}$ emissions, with improvements to the transportation infrastructures. The idea that travel time savings are not beneficial, because improving road infrastructures in cities will attract even more traffic, is not corroborated by the empirical data. Understanding the decision mechanisms underlying the individual mobility demand and the use of private vehicles in a city is a fundamental task to forecast the impact of new transportation infrastructures or of traffic restriction policies. In our opinion, we clearly need to replace the assumptions of a constant travel time budget and an induced travel demand, with new models, which should necessarily encompass both individual behaviour and city development.

\section{Methods}

\subsection{GPS database}

This work is based on the analysis of a large database of GPS measures sampling the trajectories of private vehicles in the whole Italy during May 2011. This database refers, on average, to $2 \%$ of the vehicles registered in Italy, containing traces of $128,363,000$ trips performed by 779,000 vehicles. Records are always registered at engine starts and stops and every $\approx 2 \mathrm{~km}$ during the trips (or alternatively every 30 seconds in the highways). Each datum contains time, latitude-longitude coordinates, current velocity and covered distance from the previous datum directly measured by the GPS system using data recorded (but not registered) each second. We define a trip as the transfer between two locations where the engine has been turned off. If the engine's downtime following a stop is shorter than 30 seconds, the subsequent trajectory is considered as a continuation of the same trip if it is not going back towards the origin of the first trajectory. We have performed filtering procedures to exclude from our analysis the data affected by systematic errors $(\approx 10 \%$ of data were discarded). The problems due to signal loss is critical when the engine is switched on or when the vehicle is parked inside a building. In those cases we have used the information redundancy to correct $20 \%$ of the data by identifying the starting position of one trip with the ending position of the previous one. When the signal quality is good the average space precision is of the order of $10 \mathrm{~m}$, but in some cases it can reach values up to 
30 meters or more [34]. Due to the Italian law on privacy, we have no direct information on the owners or any specific knowledge about the social characters of the drivers sample.

The GPS data base is collected for insurance reasons using black boxes installed on vehicles, whose owners agreed with a special insurance contract. As a matter of fact, these contracts are more attractive for young people or are used on fleet of vehicles. This is a bias in our sample to study human mobility, since young people may use the private vehicle in a different way with respect to elder people. However our point of view is that the universal statistical properties of human mobility discussed in the paper are not affected, due to the large number trajectories and the different urban contexts. Some vehicles present in the database belong to private companies' fleets. In this case, employers who use the car for professional reasons might show a different behaviour, but they contribute to a small percentage of all vehicles and therefore their statistical weight is small.

As the drivers's city of residence is unknown, it has been necessary to associate each car to an urban area using the available information. We have established that one driver lives in a certain city if the most part of its parking time is spent in the corresponding municipality area. For each driver, we have considered all the mobility performed in a day (inside and outside the urban area) to measure daily TTE $T$. In this way, it is possible to measure the average value of $T$ for over 1,200 different municipalities, where we have at least 100 vehicles. Moreover, for a smaller number of cities we have sufficient data to analyse the shape of the probability density $p(T)$ or of the cumulative distribution $P(T)=$ $\int_{0}^{T} p\left(T^{\prime}\right) d T^{\prime}$, as done in [20] on a similar dataset.

\subsection{A duration model for Travel-Time Expenditures}

An application of duration model to travel-time analysis has recently been proposed [35]. This type of model allows a mesoscopic description of the empirical data for a large range of human and animal temporal behaviours [36].

Using the GPS data base on single vehicle trajectories, it is possible to study the empirical TTE distribution for all cities that had at least a sample of 100 monitored vehicles (see Table S1). As example, in Figure 1 we show the TTE empirical distributions for Naples, the largest cities in the South of Italy $(\approx 1$ million inhabitants) and Grosseto, a small city in the center of Italy ( $\approx 80,000$ inhabitants). This behaviour of the TTE distribution is observed in all the considered cities. The parameter $\beta$, computed by interpolating the empirical curves (see Eq. (1)), defines the average time scale of individual daily mobility and it is a characteristic of each city. The distribution of the average TTE $\langle T\rangle$ for those cities is reported in Figure 5 together with a normally distribution with mean $1.43 \mathrm{~h}$, and standard deviation $0.15 \mathrm{~h}$. Those values are thus significantly larger than the expected TTB of $1.1 \mathrm{~h} \mathrm{[15].}$

From the comparison of definitions of TTB for different modes of transportation, bodily energy consumption rates have to be taken into account to define a universal travel-energy budget [23]. The TTB $\beta$ can be therefore interpreted as a physiological limit to daily mobility: it is the stress and fatigue accumulated during traveling that restricts the time an individual is willing to spend on mobility in a day. Let $T$ the TTE of an individual, then we can introduce the survival function $S(T)$ as the probability that the TTE is greater than $T$. Assuming the Markov properties for the evolution of $T$, we have the relation

$$
S(T+\Delta T)=[1-\lambda(T) \Delta T] S(T)+o(\Delta T),
$$



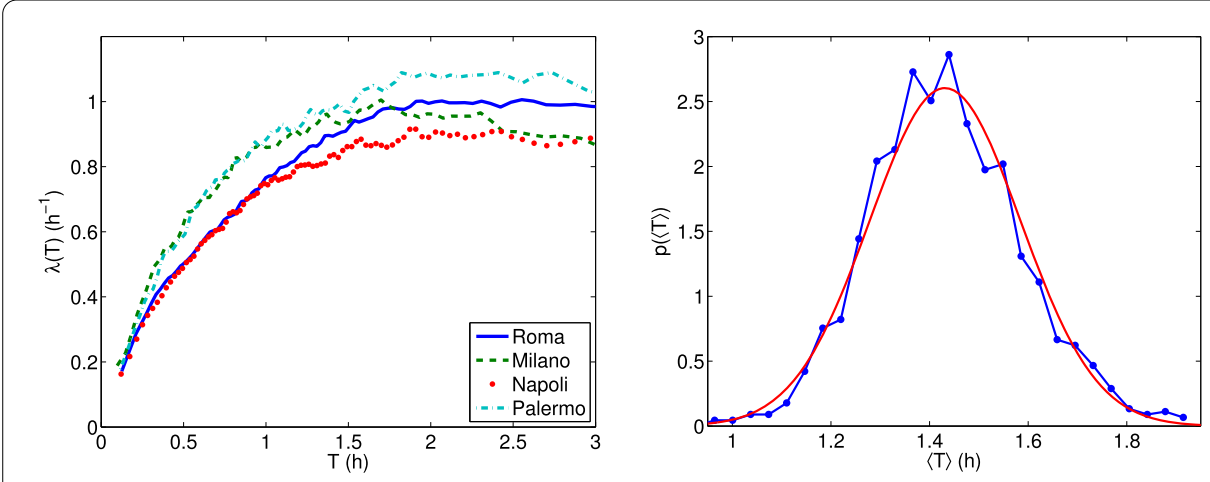

Figure 5 Variability of the Travel-Time Expenditures among cities. (Left) Different behaviours of the empirical hazard functions to model the mobility in a city. Hazard functions $\lambda(T)$ for the cities of Milan in Northern Italy with 1.3 millions inhabitants (dash line), Rome, the capital, in Central Italy with 2.7 millions inhabitants (solid line), Naples in Southern Italy 1 million inhabitants (dot line) and Palermo in Sicily 700 thousand inhabitants (dot-dash line). We have significant differences, particularly in the height of the plateau $(1 / \beta)$ that is related to the TTB. (Right) Average TTE in Italian cities. The distribution of the average TTE for 1,233 Italian municipalities where we have at least 100 GPS equipped vehicles (dots and lines) can be interpolated with a Gaussian with mean $1.43 \mathrm{~h}$ and standard deviation $0.15 \mathrm{~h}$ (solid line).

where $\lambda(T)$ is the hazard function, which is related to the conditional probability $\pi(T+\Delta T \mid T)$ to realise a TTE $T+\Delta T$ if one has spent a TTE $T$. The hazard function can be theoretically defined as

$$
\lambda(T)=\lim _{\Delta T \rightarrow 0} \frac{1-\pi(T+\Delta T \mid T)}{\Delta T} .
$$

If we consider an ensemble of individuals, the hazard function has to be empirically defined as an average value

$$
\lambda(T)=\left\langle\frac{1-\hat{\pi}(T+\Delta T \mid T)}{\Delta T}\right\rangle_{\Delta T}
$$

where $\hat{\pi}(T+\Delta T \mid T)$ refers to the conditional probability to observe a TTE $T+\Delta T$ of the individual dynamics and the average value is computed over the distribution of the possible increments $\Delta T$ in the considered population. $S(T)$ is related to the probability distribution $p(T)$ with $p(T)=-d S(T) / d T$. When the hazard function is constant, the underlying stochastic process is a stationary Poisson distribution. But the empirical hazard function, evaluated from GPS data (see Figure 5(left)), shows an exponential decay from the asymptotic uniform behaviour (see Figure S1 and Additional file 1), which can be analytically interpolated by

$$
\lambda(T)=\beta^{-1}[1-\exp (-T / \alpha)]
$$

We identify the parameter $\beta$ with the TTB, whereas $\alpha$ may represent the typical average time associated the private car mobility, since the hazard function $\lambda(T)$ is small when $T \leq \alpha$. As a matter of fact, both quantities are characteristic of a city. The timescale $\alpha$ is associated to the accessibility of desired destinations in the city [22]. Indeed, it is interpreted as the average time necessary to satisfy the mobility demand using private cars. 
Larger values of $\alpha$ mean lower accessibility. Given $\lambda(T)$, we can compute the analytic form of the TTE probability distribution by explicitly solving Eq. (2) (see Additional file 1)

$$
p(T)=\beta^{-1} \exp \left(\alpha \beta^{-1}\right)(1-\exp (-T / \alpha)) \exp \left(-\alpha \beta^{-1} \exp (-T / \alpha)-T / \beta\right) .
$$

According to Eq. (5), for $T \gg \alpha$ the dominant term is $\exp (-T / \beta)$ and we recover the exponential tail of the empirical TTE distributions. In Figure 1, we show two interpolations of the empirical distributions by using of the function (6). The associated fits for the hazard functions are displayed in Figure S1. We have found a very good agreement considering cities of different size, importance, position and infrastructure development (see Table S1).

\subsection{A time consumption model}

To interpret the empirical results on an individual level, we formulate a time consumption model where each individual progressively accumulates travel-time according to a well defined strategy. This interpretation is based on three key aspects:

(i) the TTE is effectively a measure of the consumed Energy [23] during mobility;

(ii) there is a log-time perception of the trip durations as the TTE increases [33];

(iii) the trip durations are exponentially distributed [21].

The first item refers to a Statistical Mechanics interpretation of the TTE distribution function according to a Maxwell-Boltzmann distribution. The second item means that after a TTE of $T$, the perceived additional cost of a new trip by a driver is proportional to $\Delta T / T$, where $\Delta T$ is the new additional trip duration. The logarithmic scaling is a reflection of Weber-Fechner psychophysical law [30]. It is possible that the individual perception of weariness is at the origin of this logarithmic weighting of time, which has been also proposed to explain the statistical properties of the duration of individual activities [20]. The third item is supported by empirical evidence: our data suggest that the travel-times cost $t$ for a single trip has also predominantly an exponential probability density within the range $4 \leq t \leq 60$ minutes (see Figure 4 (left))

$$
p(t) \approx\langle t\rangle^{-1} \exp (-t /\langle t\rangle)
$$

This result has been shown to be universal across different cities, with the characteristic decaying time $\langle t\rangle$ growing with city population [21]. In Additional file 1, we show that $\langle t\rangle$ also varies among the considered cities and might depend upon house prices, city surface and average travel speeds (see Figure S2). In our model, each individual progressively accumulates travel-time to determine his TTE. According to our first assumption, a driver will accept a TTE of $T$ with a probability

$$
P(T)=\exp \left(-\frac{T}{\bar{\beta}}\right)
$$

where $\bar{\beta}$ is the characteristic TTB of the population. Then the individual conditioned probability to accept a new trip of duration $\Delta T$ after a TTE of $T$ is written

$$
\hat{\pi}(T+\Delta T \mid T)=\frac{P(T+\Delta T)}{P(T)} .
$$


However the $\Delta T$ distribution for the new trip is not independent from the elapsed TTE $T$ since users are reluctant to accept long trips when the TTE exceeds $\bar{\beta}$. Then we define a conditional $\Delta T$ distribution, which takes into account the elapsed TTE, by using a threshold function $\theta_{a}(x)$

$$
\theta_{a}(x)= \begin{cases}1 & \text { if } x<a \\ 0 & \text { otherwise }\end{cases}
$$

According to our assumptions, the distribution (7) is substituted by the conditional distribution

$$
p(\Delta T \mid T) \approx\langle t\rangle^{-1} \exp (-\Delta T /\langle t\rangle) \theta_{a}(\Delta T / \gamma),
$$

where the threshold $a$ and the time scale $\gamma$ depend on $T$ or on other individual features. The parameter $a$ is the acceptability threshold for a new trips, whereas $\gamma$ defines the perceived measure unit of the cost of the new trip. The empirical observations suggest that the threshold $a$ depends on the average number of activities $\langle n\rangle$ of an individual. This is illustrated by the correlation between the mobility timescale $\alpha$ (see Eq. (5)) divided by $\langle n\rangle$ and $\langle t\rangle$ (see Figure 6(left)). To define $\gamma$, we assume a logarithmic perception of the trip time cost so that $\gamma \propto T$. Then we set the threshold $a=x_{\max } /\langle n\rangle$ and $\gamma=T$, so that the threshold function is written in the form

$$
\theta_{x_{\max } /\langle n\rangle}\left(\frac{\Delta T}{T}\right)= \begin{cases}1 & \text { if } \frac{\Delta T}{T}<x_{\max } /\langle n\rangle, \\ 0 & \text { otherwise }\end{cases}
$$

where $x_{\max }$ turns out to be an universal threshold. Therefore Eq. (10) is based on the assumption that the propensity of a driver to accept a further trip of duration $\Delta T$ after having performed a TTE of $T$, scales as $\langle n\rangle / T$, where $\langle n\rangle$ is the average number of daily
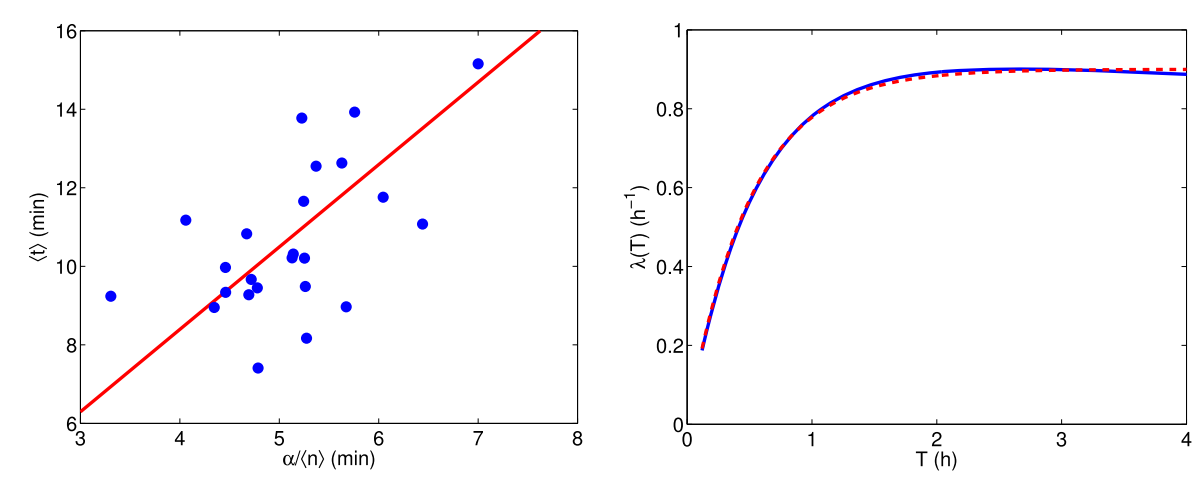

Figure 6 Validation of the time consumption model. (Left) Correlation between the time of a single trip duration $\langle t\rangle$ and the ratio between the short travel-time expenditures and the average number of activities $\alpha /\langle n\rangle$ : each dot refers to a different city $(r=0.57)$; the straight line has a slope $x_{\max }=\langle t\rangle\langle n\rangle / \alpha=2.1 \pm 0.1$ (error is s.e.m.). (Left) Comparison between the hazard function (5) inferred from our empirical data and the hazard function computed using the decision model: the hazard function derived from the decision model (solid blue line), using realistic parameters values $\langle t\rangle \simeq 0.2 \mathrm{~h}, \bar{\beta}=1 \mathrm{~h},\langle n\rangle=5$ and $x_{\max }=2$ is compared with the empirical hazard function (5) (dashed red line) with $\beta=1.08 \times \bar{\beta}$ and $\alpha \simeq 0.5 \mathrm{~h}$. 
activities. In other words the individuals that perform more trips using private vehicles have a greater TTB: this could be also a consequence of the multi-modal mobility, which is not included in our database, and that allows individuals to divide their TTB according the different transportation means used. Moreover, an individual seems to organise the mobility using the TTB as a mobility energy (with the constraint of performing the compulsory daily activities), but keeping the percentage of TTE fluctuations constant. Using empirical values for the different quantities in the relation (11) we can estimate $x_{\max } \simeq 2$ (see Figure 6(left)).

We compute the empirical hazard function for a population of drivers according to the definition (4)

$$
\lambda(T)=\langle t\rangle^{-1} \int_{0}^{\infty} \frac{1-\exp (-\Delta T / \beta)}{\Delta T} \theta_{x_{\max } /\langle n\rangle}\left(\frac{\Delta T}{T}\right) \exp (-\Delta T /\langle t\rangle) d \Delta T .
$$

An explicit calculation (see Additional file 1) shows that the hazard function of the model has the same analytic form as the empirical interpolation (5), where the timescale of the short TTE under-expression is

$$
\alpha \simeq\langle n\rangle\langle t\rangle / x_{\max }
$$

as one can see in Figure 6(right).

\section{Additional material}

Additional file 1: Supplementary information (pdf)

Competing interests

The authors declare that they have no competing interests.

Authors' contributions

$R G, A B$ designed and performed research and wrote the paper; $R G, A B$, SR performed research. $R G$ prepared the figures.

\section{Author details}

${ }^{1}$ Institut de Physique Théorique, CEA-Saclay, Gif-sur-Yvette, France. ${ }^{2}$ Department of Physics and Astronomy, University of Bologna, Bologna, Italy. ${ }^{3}$ INFN Bologna section, Bologna, Italy.

\section{Acknowledgements \\ We thank D. Helbing for useful comments on an early draft. We thank Octo Telematics S.p.A. for providing the GPS database. RG thanks M. Barthelemy, G. Carra, Y. Crozet, M. Lenormand, T. Louail and R. Louf for useful discussions at the QuantUrb seminars.}

Received: 6 July 2015 Accepted: 26 October 2015 Published online: 04 November 2015

\section{References}

1. González MC, Hidalgo CA, Barabási AL (2008) Understanding individual human mobility patterns. Nature 453:779-782

2. Song C, Koren T, Wang P, Barabási A-L (2010) Modelling the scaling properties of human mobility. Nat Phys 6:818-823

3. Cheng Z, Caverlee J, Lee K, Sui DZ (2011) Exploring millions of footprints in location sharing services. In: ICWSM-11, pp 81-88

4. Noulas A, Scellato S, Lambiotte R, Pontil M, Mascolo C (2012) A tale of many cities: universal patterns in human urban mobility. PLOS ONE 7(5):e37027

5. Vespignani A (2012) Modelling dynamical processes in complex socio-technical systems. Nat Phys 8:32-39

6. Hawelka B, Sitko I, Beinat E, Sobolevsky S, Kazakopoulos P, Ratti C (2014) Geo-located Twitter as proxy for global mobility patterns. Cartogr Geogr Inf Sci 41(3):260-271

7. Lenormand M, Picornell M, Cantù-Ros OG, Tugores A, Louail T, Herranz R, Barthelemy M, Frías-Martínez E, Ramasco JJ (2014) Cross-checking different sources of mobility information. PLOS ONE 9(8):e105184

8. Axhausen KW (2008) Social networks, mobility biographies, and travel: survey challenges. Environ Plan B 35:981-996

9. Zahavi Y (1974) Traveltime budget and mobility in urban areas. US Department of Transportation, Washington, DC 
10. Stopher PR, Zhang Y (2011) Travel time expenditures and travel time budgets - preliminary findings. Working paper ITLS-WP-11-04, Institute of Transport and Logistics Studies, The University of Sydney

11. Marchetti C (1994) Anthropological invariants in travel behavior. Technol Forecast Soc Change 47:75-88

12. Kung KS, Greco K, Sobolevsky S, Ratti C (2014) Exploring universal patterns in human home-work commuting from mobile phone data. PLoS ONE 9:e96180

13. Mokhtarian PL, Chen C (2004) TTB or not TTB, that is the question: a review and analysis of the empirical literature on travel time (and money) budgets. Transp Res, Part A 38:643-675

14. Cervero R (2011) Beyond travel time savings: an expanded framework for evaluating urban transport projects. Transport Research Support, World Bank/DFID

15. Metz D (2008) The myth of travel time saving. Transp Rev 28:321-336

16. Toole-Holt L, Polzin SE Pendyala RM (2005) Two minutes per person per day each year: exploration of growth in travel time expenditures. Transp Res Rec 1917:45-53

17. van Wee B, Rietveld P, Meurs H (2006) Is average daily travel time expenditure constant? In search of explanations for an increase in average travel time. J Transp Geogr 14:109-122

18. Milthorpe $F(2007)$ Consistency in daily travel time - an empirical assessment from Sydney travel surveys. In: Proceedings of the 30 th Australasian transport research forum

19. Louf R, Barthelemy M (2014) How congestion shapes cities: from mobility patterns to scaling. Sci Rep 4:5561

20. Gallotti R, Bazzani A, Rambaldi S (2012) Toward a statistical physics of human mobility. Int J Mod Phys C 23:1250061

21. Gallotti R, Bazzani A, Rambaldi S, Barthelemy M (2015) How transportation hierarchy shapes human mobility. arXiv:1509.03752

22. Hansen W (1959) How accessibility shapes land use. J Am Inst Plann 25:73-76

23. Kölbl R, Helbing D (2003) Energy laws in human travel behaviour. New J Phys 5:48.1-48.12

24. Bazzani A, Giorgini B, Rambaldi S, Gallotti R, Giovannini L (2010) Statistical laws in urban mobility from microscopic GPS data in the area of Florence. J Stat Mech Theory Exp 2010:P05001

25. Liang X, Zheng X, Lv W, Zhu T, Xu K (2012) The scaling of human mobility by taxis is exponential. Physica A 391:2135-2144

26. Liang X, Zhao J, Dong L, Xu K (2012) Unraveling the origin of exponential law in intra-urban human mobility. Sci Rep 3:2983

27. Bettencourt LMA, Lobo J, Helbing D, Künert C, West GB (2007) Growth, innovation, scaling, and the pace of life in cities. Proc Natl Acad Sci USA 104:7301-7306

28. Yan X-Y, Han X-P, Wang B-H, Zhou T (2013) Diversity of individual mobility patterns and emergence of aggregated scaling laws. Sci Rep 3:2678

29. Gallotti R (2013) Statistical physics and modeling of human mobility. PhD thesis, University of Bologna, Italy

30. Dehaene S (2003) The neural basis of the Weber-Fechner law: a logarithmic mental number line. Trends Cogn Sci 7:145-147

31. Domencich T, McFadden D (1975) Urban travel demand: a behavioral analysis. North-Holland, Amsterdam

32. Takahashi T, Hidemi O, Radford MHB (2008) Psychophysics of time perception and intertemporal choice models. Physica A 387:2066-2074

33. Helbing D (2010) Quantitative sociodynamics. Springer, Berlin

34. Bazzani A, Giorgini B, Giovannini L, Gallotti R, Marchioni M, Rambaldi S (2011) Towards congestion detection in transportation networks using GPS data. In: IEEE international conference on privacy, security, risk, and trust, and IEEE international conference on social computing

35. Juan Z, Xianyu J (2010) Daily travel time analysis with duration model. J Transp Syst Eng Inf Technol 10:62-67

36. Proekt A, Banavar JR, Maritan A, Pfaff DW (2012) Scale invariance in the dynamics of spontaneous behavior. Proc Natl Acad Sci USA 109:10564-10569

\section{Submit your manuscript to a SpringerOpen ${ }^{\circ}$ journal and benefit from:}

- Convenient online submission

Rigorous peer review

- Immediate publication on acceptance

- Open access: articles freely available online

- High visibility within the field

- Retaining the copyright to your article 